\title{
Ein gerichtsmedizinisches toxikologisches Gutachten des Zürcher Stadtarztes Dr. Johann Scheuchzer aus dem Jahr 1737
}

\author{
Von Bernhard Milt, Zürich
}

Am 18. Juli des Jahres 1737 erhielt der Zürcher Stadtarzt Dr. med. J. Scheuchzer vom damaligen Kyburger Landvogt H. J. Leu den Auftrag zu einem gerichtsmedizinischen Gutachten. Im hochgelegenen Dörflein Dürstelen im Zürcher Oberland, an der Straße von Hittnau nach Bauma, war ein einundsechzigjähriger Mann, der Landwirt Hans Schneyder, unter verdächtigen Umständen gestorben. Man munkelte von Giftmord; eine Hausdurchsuchung war bereits vorgenommen worden, und die Frau des Toten saß in Sicherheitshaft. Der Tatbestand, der den Landvogt zu einer Untersuchung zwang, war folgender:

Vom Hittnauer Pfarrer Heınrich Fäsi, einem achtundvierzigjährigen und seit fünfzehn Jahren in der Gemeinde amtenden Geistlichen, war der Bericht eingegangen, er habe eine Bestattung des in Dürstelen verstorbenen und in Hittnau kirchgenössigen Landwirts verweigert, weil der Zustand der Leiche seinen Verdacht erweckt habe, es könnte ein außergewöhnlicher Todesfall vorliegen. Die Leiche war bereits nach Hittnau übergeführt gewesen und lag auf dem Totenbaum zur Bestattung bereit, als der Pfarrer ihre Heimschaffung anordnete. Obschon nichts von einer vorgängigen Schlägerei bekannt war, war ein Finger der Leiche angeblich blutbefleckt. Noch immer trat aus Mund und Augenwinkeln eine blutig aussehende Flüssigkeit.

Landvogt LEU hatte diesen Bericht etwas verspätet erhalten, da er gerade abwesend war. Der Tod war am 3. Juli eingetreten. Am 6. Juli beorderte er den Winterthurer Arzt Dr. Kronauer nach Dürstelen, damit dieser die Leiche eingehender inspiziere. Ohne Auftrag nahm dieser an Ort und Stelle auch gleich eine Obduktion vor. Da er drei verschiedene Pulver im Haus vorfand, nahm er Proben davon mit, da er den Verdacht hegte, es handle sich um einen Vergiftungstod. Von einem dieser Pulver gab er zu Hause einer Katze zu fressen, die bald nachher starb, was seinen Verdacht natürlich bestärkte. So erstattete er Landvogt LEU seinen Bericht mit dem Verdacht, es liege eine Vergiftung vor, voraussichtlich mit Quecksilbersublimat und weißem Arsen oder Rattengift. Daraufhin nahm 
der Landvogt die Frau des Verstorbenen in Sicherheitshaft und ordnete er eine Hausdurchsuchung an. Was hatte Dr. Kronauer festgestellt?

Die von ihm inspizierte Leiche hatte ein schauderhaftes Aussehen gezeigt. Der Kopf war dick angeschwollen und die Augen standen weit vor den Höhlen. Die Zunge war unförmig dick, schwarzbraun und ragte weit zum Mund hinaus. Aus Mund und Augenwinkeln quoll eine braune Flüssigkeit von blutigem Aussehen. Wo sie mit der Haut in Berührung kam, ulzerierte sie dieselbe, in der Halsgrube bis tief in die Muskulatur hinein. Eine Sonde aus Silber verfärbte sich darin gelbschwarz. Hals, Brust, Bauch und Geschlechtsteile waren zum Platzen gebläht und angeschwollen. Der Enddarm ragte weit zum After hinaus und die ganze Haut war schwärzlich verfärbt. Nach Durchtrennung von Bauchdecken und Bauchfell drangen die braunroten Därme mit lautem Geräusch und fürchterlichem Gestank durch die Öffnung, stark gebläht, aber leer wie der Magen. Nur an der Innenwand klebte eine bläuliche, bleifarbene Masse. Die Schleimhaut von Magen und Speiseröhre schien erheblich verdickt, und im linken Magenfundus fand sich ein Loch, das für den Zeigefinger bequem durchgängig war, gerade gegenüber der Milz. Diese war schwarz und zerfiel bei der geringsten Berührung. Weitere geschwürige oder gar eitrige Prozesse hatten sich im Magen-Darm-Tractus nicht gefunden. Auch die Brusthöhle wurde näher untersucht, ohne Befundangabe. Auf eine Inspektion von Schädelhöhle und Gehirn glaubte der Arzt verzichten zu dürfen.

Dr. Kronauer hatte im Haus des Toten drei verschiedene Pulver vorgefunden, von denen ihm aber nur eines verdächtig vorkam. Von diesem gab er, zusammen mit Fleisch, seiner Katze zu fressen, die bald darauf ihre Eßlust verlor, gesträubte Haare zeigte und jämmerlich zu heulen begann. Ihre Exkremente wurden blutig; sie begann zu zittern und konnte sich bald nicht mehr auf den Beinen halten. Kurz darauf starb sie. Ihr Kopf war dick angeschwollen, besonders die Augenlider, die sie nicht mehr hatte öffnen können. Die anschließende Sektion ergab Geschwüre im MagenDarm-Kanal. Das Krankheitsbild schien mit demjenigen des Verstorbenen Ähnlichkeit zu zeigen. Dieser war mit einem Schüttelfrost erkrankt und hatte ebenfalls stark gezittert und geschlottert. Außer über heftigen Durst hatte er über starke Magen- und Leibschmerzen geklagt und raschen Kräftezerfall gezeigt. So kam Dr. Kronauer zum Schluß, der Landwirt sei einer Vergiftung erlegen, vermutlich eben mit Quecksilbersublimat und Rattengift oder weißem Arsen.

Der Sache weniger sicher schien der Landvogt zu sein. Am 18. Juli 
übersandte er dem Zürcher Stadtarzt Dr. Schevchzer den ärztlichen Bericht, Proben der drei Pulver und eine kurze Schilderung des Falles, und schon fünf Tage später war er im Besitz von dessen Gutachten. Darin waren Untersuchungsergebnisse und Bericht von Dr. KRoNauer in wenig kollegialer Weise oft geradezu höhnisch zerzaust und lächerlich gemacht. Dem Sektionsbefund maß er nicht die mindeste Bedeutung zu, da daraus lediglich hervorgehe, daß sich die Leiche bereits im Zustand erheblicher Zersetzung befunden habe. Da es sehr heißes Sommerwetter, der Tote zweimal transportiert worden und in Hittnau auf dem Totenbaum längere Zeit der Sonnenbestrahlung ausgesetzt gewesen war, kam das dem Stadtarzt nicht außergewöhnlich vor. Nach seiner Meinung hatte Dr. Kronauer aus dem Obduktionsbefund völlig falsche Schlüsse gezogen. Vollends das Katzenexperiment entbehrte in seinen Augen als einzige Untersuchungsmethode jeder Beweiskraft, da es hinlänglich bekannt sei, daß es Substanzen gebe, die für Tiere zwar giftig seien, nicht aber für Menschen, und umgekehrt. Dr. Kronauer hatte zwar angegeben, er habe die Pulver auch im Feuer untersucht und bei dem von ihm inkriminierten gefunden, daß es stark ätzend wirke, nicht nur auf den Körper, auch auf Metalle, ohne aber seine Erfahrungen mitzuteilen, die einen solchen Schluß erlaubten. Der Stadtarzt fand, sein Winterthurer Kollege habe alle seine Verdachtsmomente auf haltlose Spekulationen basiert.

Dr. Johann Scheuchzer, der jüngere Bruder des bekannteren JohanN Јаков Sche uchzer, war diesem als Zürcher Stadtarzt, Chorherr und Professor Physicae an der Stiftsschule im Amte nachgefolgt und bekleidete diese Stelle seit etwa drei Jahren, nachdem er vorher in einem abenteuerreichen Leben Soldat in fremden Diensten, Reisebegleiter und Naturforscher, Kaufmann, Ingenieuroffizier im Krieg von 1712, Bibliothekar in seiner Vaterstadt und Landschreiber in Baden gewesen war. Nach unglaublich kurzem Studium war er im Jahr 1705 Dr. med. in Basel geworden. Mit vierundzwanzig Jahren war er bereits Mitglied der kaiserlich Leopoldinischen Akademie. Seine Agrostographie gehört zu den klassischen Werken der botanischen Weltliteratur und begründete die Gräserkunde. Nicht weniger begabt als sein Bruder, stand er gleichwohl stets in dessen Schatten; bis heute hat er es zu keiner eingehenderen biographischen Würdigung gebracht, obgleich der noch vorhandene Briefwechsel mit seinem Bruder reichen Stoff liefern würde. Das Gutachten des Stadtarztes umfaßt in der Abschrift im Gschaubuch (Sign. H I 322 des Zürcher Staatsarchivs) 18 Seiten, die gesamte Korrespondenz ihrer 36, Seite 330-366. 
Sche uchzer schritt nun seinerseits zur Untersuchung des fraglichen Pulvers. $\mathrm{Zu}$ Vergleichszwecken wiederholte er das Katzenexperiment, in der Meinung, Winterthurer und Zürcher Katzen würden sich wohl nicht allzusehr voneinander unterscheiden. Obschon er seinem Versuchstier eine tüchtige Dosis davon verabreichte, verlor es seine Eßlust nicht, sträubten sich nicht einmal seine Haare, zitterte und starb es nicht; es fühlte sich im Gegenteil ganz wohl. Die verschiedenen Pulver mit der Zunge auf ihren Geschmack zu prüfen, trug Scheuchzer nicht die geringsten Bedenken. Dann schritt er zu ihrer chemischen Begutachtung.

Er streute vom Pulver, das angeblich weißen Arsen und Quecksilbersublimat enthalten sollte, eine Portion auf glühende Kohlen. Dabei bemerkte er weder eine Rauch- noch Dampfentwicklung; es zeigten sich lediglich explodierende leuchtende Fünklein, die auf das Vorhandensein von Salpeter schließen ließen (pulvis ardentibus prunis injectus nullum plane fumum emittit nec proin metallum dealbat, sed in prunis igniculos lucidos detonantis nitri indices conspiciendos praebet). Er verglich damit das Verhalten von Quecksilbersublimat und Arsen unter gleichen Bedingungen. Streute er Quecksilbersublimat auf glühende Kohlen, entwickelte sich sofort ein weißlicher Rauch, der Messing mit einem sehr feinen, weißlich-aschenförmigen Staub überzog; in das Messing eingerieben, entfärbte er dasselbe wie lebendiges Quecksilber (Mercurius sublimatus prunis injectus fumum emittit illico albicantem, Orichalcum polline subtilissimo ex albido cinerescente inficiens qui Orichalco affrictus illud Mercurii vivi instar dealbat et eidem argenteum colorem conciliat). Messing nahm also an der betreffenden Stelle Silberfarbe an. Scheuchzer kannte somit die Reduktionsprobe, wenigstens in ihren Wirkungen. Irrte man zu jener Zeit auch in den Ansichten über Natur und Wesen dieses Elements, so waren doch seit dem Mittelalter viele seiner Verbindungen bekannt. Auf gleiche Weise nahm der Stadtarzt die Reduktionsprobe des weißen Arsens, der arsenigen Säure, vor. Streute er von dieser Substanz auf glühende Kohlen, entstand ebenfalls ein Rauch mit einem zum Brechen reizenden Knoblauchgeruch. Dieser Rauch bildete auf Messing einen zuerst weißen und dann schwarz werdenden Überzug, der, wenn man ihn auf Messing rieb, dessen Farbe nicht veränderte (Arsenicum album prunis injectum fumum similem emittit, sed allii odorem nauseabundum spirat et fumus idem Orchialcum albo et hinc inde nigricante polline inficit qui Orichalco affrictus nullam eidem albidinem communicat). Da die Kohle der arsenigen Säure Sauerstoff entzieht, entsteht auf diese Weise Arsensuboxyd und Arsen, wobei 
die Bildung von Arsensuboxyd diesen Knoblauchgeruch bedingt. Das Faktum an sich war Sche UCHZER bekannt, wenn auch ohne zutreffende Kenntnis der chemischen Voraussetzungen. Diese Proben sprachen auf jeden Fall nicht dafür, daß sich im inkriminierten Pulver Quecksilbersublimat oder Rattengift befand. Scheuchzer begnügte sich aber nicht mit dieser Probe und machte eine weitere.

Er brachte vom fraglichen Pulver eine Portion in ein Gemisch von Aquafort und Wasser, Aquafort gewann man durch Destillation von Vitriol und Salpeter. Er bemerkte ein zischendes Aufbrausen und eine Vermischung des Pulvers mit der Lösungsflüssigkeit, unter Bildung eines weißen Sediments, das bald wie weißer Schwefel an der Oberfläche schwamm. Ganz anders verhielten sich Quecksilbersublimat und weißer Arsen. Beim erstern kam es zu keinem Aufbrausen und kaum zu einer Vermischung mit dem Lösungsmittel, die nur «aegre et non nisi violenta cum conquassatione» erreicht werden konnte. Das Pulver sank als weißes Sediment auf den Grund. Fügte er kohlensaures Kali (Oleum tartari per deliquium) bei, verfärbte sich das inkriminierte Pulver in keiner Weise, während das Quecksilbersublimat orangefarben wurde, «egregium colorem aurantiorum» annahm. Auch hier war dem Stadtarzt die Herstellung des gelben oder roten Präzipitats als Quecksilberverbindung bekannt, ohne daß er von Quecksilberoxyd etwas wissen konnte. Auch der weiße Arsen bewirkte in der Lösung von Aquafort und Wasser kein Aufbrausen, noch vermischte er sich mit ihr, nahm aber mit kohlensaurem Kali, mit Oleum tartari per deliquium keine andere Farbe an. Fügte man aber noch Quecksilbersublimat bei, trat keine rotgelbe, sondern eine dunkle Verfärbung auf, so daß sich auch ein Gemisch von Rattengift und Quecksilbersublimat deutlich vom Verhalten des fraglichen Pulvers unterschied. Die letztere Probe wurde damals ziemlich häufig angewandt, weil man völlig zu Unrecht vermutete, das in den Handel gebrachte Quecksilbersublimat werde von den Händlern häufig mit arseniger Säure vermischt, zur leichteren Sublimierbarkeit und vor allem zur Erzielung eines höhern Gewichts. Trat bei dieser Probe eine Dunkelfärbung ein, rührte sie weit eher von einer Vermischung mit Kalomel als von Arsen her.

Nach diesen Untersuchungen kam der Stadtarzt vollends zur Überzeugung, der von Dr. Kronauer geäußerte Verdacht, das untersuchte Pulver bestehe aus Rattengift und Quecksilbersublimat, beruhe lediglich auf haltlosen Spekulationen und sei völlig aus der Luft gegriffen; für ihn sprach überhaupt nichts für die Giftigkeit dieses Pulvers. Er war überzeugt, daß 
die inhaftierte Witwe des Verstorbenen leicht darüber Aufschluß geben könnte, woher diese Pulver stammten, wozu sie gebraucht wurden und weshalb sie dieselben aufbewahrt habe.

Das fragwürdige Pulver hielt er für ein in jeder Apotheke käufliches und in jeder Pharmakopoe erwähntes Pulvis temperans, bestehend aus Salpeter, Krebssteinen und Antimonium diaphoreticum. Es sei geeignet, die Transpiration in Gang zu bringen, innere Hitze zu dämmen und werde auch von Stadtarzt Landolt sehr empfohlen. Im zweiten, roten Pulver glaubte er das bekannte Pulvis antispasmodicus zu erkennen, aus Salpeter und Cinnabaris Antimonii zusammengesetzt, das als nervenberuhigendes Heilmittel in Ansehen stehe. Einzig über das dritte Pulver wollte er sich nicht näher äußern, weil ihm zu kleine Versuchsmengen zur Verfügung gestellt worden seien; offensichtlich handle es sich um gepulverte Wurzeln. Auch bei diesem nahm er an, die Witwe wüßte wahrscheinlich Bescheid.

Um sich über den Todesfall ein etwas zutreffenderes Bild machen zu können, verlangte der Stadtarzt vor allem nähere Angaben über das Krankheitsgeschehen selber: Wie lange hatte die Krankheit gedauert; wie und wann hatte sie angefangen und woher war sie dem Patienten zugestoßen; welches war der Verlauf von Tag zu Tag; was wußten andere Hausbewohner oder gelegentliche Besucher über ihre Eindrücke und Erfahrungen zu erzählen ? Objektive physikalische und chemische Untersuchungsmethoden gab es zu jener Zeit noch nicht; jede Diagnose wurde lediglich aus Anamnese, äußerm Erscheinungsbild und Verlauf der Krankheit gestellt.

Landvogt LEU beeilte sich, diese Fragen zu beantworten. In Kyburg vernahm er die Witwe und in Dürstelen verschiedene weitere Personen, vor allem den an einem ähnlichen Krankheitsbild noch immer leidenden Sohn und dessen Frau, sowie verschiedene gelegentliche Besucher, Verwandte und Freunde des Toten. Aus diesen Aussagen ergab sich folgendes:

Die Pulver stammten von einem «landstreichenden Krämer», gegen den aber nichts Nachteiliges vorlag. Ähnliche Erkrankungsformen waren damals in der Gegend gehäuft aufgetreten; man hatte sie auf den Genuß von verdorbener Milch zurückgeführt. Der Tote hatte am Abend des 27. Juni über schwere Beine und Brennen in der Herzgegend geklagt und am folgenden Morgen, als er aufstehen wollte, einen Schüttelfrost erlitten. Er zeigte dann hohes Fieber, klagte über heftiges Brennen in der Herzgegend, hatte einen quälenden, trockenen Husten und litt unter entsetzlichem Durst. Alle halbe Stunden verlangte er nach möglichst kaltem 
Wasser oder kalter Milch, direkt aus dem Keller. Er aß beinahe nichts mehr, trank nur noch etwas Wein. Außerdem zeigte er Stuhl- und Urinverhaltung. Später traten dann offenbar noch Magen- und Darmkrämpfe hinzu. Rasch verlor er seine Kräfte. Am 30. Juni fiel eine gewisse Euphorie auf. Husten und Brennen ließen in der Folge etwas nach, so daß der Patient am 3.Juli von einer Besserung sprach. Am Abend fand ihn aber ein Besucher im Delirium, und um zehn Uhr verschied der Kranke unerwartet. Ein Arzt war nicht zugezogen worden.

Schon am Krankenbett und erneut nach dem Tod war zwischen der Witwe und dem in Hausgemeinschaft wohnenden verheirateten Sohn ein Kampf um die Hinterlassenschaft ausgebrochen, und der Sohn verdächtigte seine Mutter in seinen Aussagen in jeder Weise. Er war wohl auch die Quelle des entstandenen Verdachts. Wenige Tage nach dem Vater war der Sohn unter ganz ähnlichen Erscheinungen erkrankt. Er beschuldigte seine Mutter, diese hätte ihm verdorbene, «bittere und ganz räße» Milch gegeben, als er durstig aus dem Wald zurückgekommen sei; nachher sei er erkrankt. Auch er hatte hohes Fieber und quälenden Husten gehabt, mit blutig-eitrigem Auswurf und heftigem Seitenstechen. Für ihn wurde ein Arzt zugezogen, der Milchdiät und Arzneien verordnete. Auf die anfängliche Verstopfung folgten heftige Durchfälle, die natürlich auch durch vom Arzt verabreichte Abführmittel herbeigeführt sein konnten.

Die Sektion war am 6.Juli, drei Tage nach dem Eintritt des Todes, um die Mittagszeit vorgenommen worden, und am selben Abend hatte man den Toten der Gruft übergeben.

In seinem Schlußbericht bemerkte Dr. Scheuchzer, daß er aus diesem etwas mangelhaften Krankheitsbericht sich zu einer sichern Diagnose nicht berechtigt fühle. Auf jeden Fall fehle nach wie vor jeder vernünftige Grund, eine Vergiftung oder auch sonst ein Verbrechen anzunehmen. Sicher habe es sich um eine akute fieberhafte Erkrankung gehandelt, beim Vater und Sohn wohl um dieselbe, offenbar mit Peripneumonie, beim Sohn auch mit Pleuritis verbunden. Die Leibschmerzen des Vaters könne man auf eine Inflammation des Magen-Darm-Tractus zurückführen, die wahrscheinlich durch den unsinnigen Genuß von kalten Getränken verursacht worden sei; besser hätte man den Alten zum Schwitzen gebracht. Das ganze Gerichtsverfahren wäre in seinen Augen völlig unnötig gewesen, wenn Dr. KroNAUERS Untersuchung etwas sachverständiger durchgeführt worden wäre. Dieser Ansicht hat sich offenbar auch der Landvogt angeschlossen und das Verfahren wohl eingestellt. 
Schon im Jahr darauf ist nach nur gut vierjähriger Tätigkeit auch Dr. Johann Scheuchzer, erst vierundfünfzigjährig, gestorben und im Kreuzgang des Großmünsters ehrenvoll bestattet worden. 